\title{
Correspondence
}

Editor: Greg Wilkinson

Contents: Supportive psychotherapy: a contradiction in terms?/Screening for HIV/Therapeutic factors in in-patient psychotherapy groups/Assaults on staff by psychiatric in-patients/Psychotherapy and dysmorphophobia/ECT for depression in dementia/ Dangerous delusions: violence and the misidentification syndromes/The need to compare the effectiveness of antidepressant drugs: in which patient populations?/Barking mad/The dopamine hypothesis/ Imipramine versus phenelzine in melancholias and dysthymic disorders/The motor disorders of severe psychiatric illness: a conflict of paradigms/Quinine psychosis/Bulimia nervosa in an atypical setting: case report from Nigeria/Catatonia in a 90 -year-old patient after depot pipothiazine injection/Mania following bereavement in a mentally handicapped man/ Psychiatric Munchausen's syndrome: a College register?/Migraine, insomnia and reactive depression due to brain serotonin deficiency?

\section{Supportive Psychotherapy: A Contradiction in Terms?}

SIR: I am writing in response to Dr Crown (Journal, February 1988, 152, 266-269). I would like to suggest that no therapy of any kind can start unless some sort of 'working alliance' or 'secure base' is first established. If one can be it is, ipso facto, supportive and an essential ingredient of dynamic psychotherapy. There is no paradox, only a puzzle that one could be perceived.

\section{Stockwell Centre Practice \\ $10 B$ Northgate Street \\ Colchester, Essex CO1 1HA}

\section{J. LuCAS}

SIR: Sidney Crown's interesting article reached a surprising conclusion. Surely all psychotherapies are, or should be, supportive? Psychotherapy is a broad term which encompasses treatments ranging from psychoanalysis to cognitive and behavioural therapies and action techniques such as Gestalt and psychodrama. The difference between the so-called 'supportive' psychotherapy and 'dynamic' psychotherapy is not in the supportive aspect, but in the aim of therapy. In the former, this is to strengthen and supplement existing defence mechanisms, leaving the unconscious alone and concentrating, as he says, on symptoms and on the present time. In dynamic psychotherapy, the aim is to make fundamental changes in these mechanisms, which involves understanding how they have arisen, on the part of the patient, through his unconscious being made conscious. The mobilising of negative emotions mentioned by Dr Crown can only be done effectively in the context of a supportive relationship. What patient would risk acknowledging his more negative side in a relationship felt to be unsupportive?

Perhaps we could replace the word 'supportive' by another such as 'maintaining' or 'non-challenging' or 'non-interpretative'?

As to the specific technical interventions as opposed to non-specific factors, Buckley et al found that interpretation and insight were key factors in symptom alleviation and positive character change, and that technical procedures related to the management of transference phenomena were critical to a positive outcome. They concluded that specific technical procedures are "central to a positive therapeutic change resulting from psychoanalysis or intensive psychotherapy, provided that they are implemented within the context of what the patient experiences as a positive therapeutic relationship".

SOPHIA HARTLAND

St Ann's Hospital

Nottingham

\section{Reference}

Buckley, P., Karasu, T. B. \& Charles, E. (1981) Psychotherapists view their personal therapy. Psychotherapy Theory Research and Practice, 18, 299-305.

SIR: I would not argue with the bulk of what Dr Crown has to say in describing dynamic and supportive psychotherapies, but I would see these as a continuum rather than separate entities. Clinical practice does not generate many patients whose treatment fits fully into one of these categories. In an individual patient the bias may be towards dynamic work where interpretations are frequent, defences challenged, 
and transference encouraged, or a supportive approach in which interpretations are rare, defences bolstered, and a more reality-based therapist-patient relationship established; this will vary also within an individual patient over time. It is appropriate on occasion for judicious, hopefully mutatative dynamic work to be done with patients whom one has largely supported; likewise, it may be appropriate as a phase in therapy to relax exploratory work in favour of support in those patients to whom one is offering dynamic psychotherapy. Beyond that the bias may change from episode to episode - a patient may be supported on an occasion of crisis, only to be re-referred a few years later for more definitive work.

By defining dynamic and supportive psychotherapies too rigidly I believe we diminish their potential. While not decrying the merits of brief focused psychotherapy, there is always a danger of writing the script of treatment in advance, which is surely counter to the 'dynamic' of dynamic psychotherapy. By sticking too rigidly to the limited goals of supportive psychotherapy we may deny our patients opportunities for growth. We are greatly indebted to Dr Crown, however, for once again emphasising that we must continue to think about what we are doing, why and how we are doing it, and how often, in psychotherapy.

Murray Royal Hospital

Perth PH2 7 BH

SIR: I first express my gratitude to Dr Crown for his stimulating paper. The article was (almost certainly) a response to an all-too-vague and inadequately documented phenomenon. In comparing dynamic and supportive psychotherapies, he dealt very briefly with comparisons concerning goals or desirable outcomes, and I believe that this area contains essential differences worth noting. Dynamic therapy is concerned with change - often very basic change of life strategy. Supportive therapy does not pursue such fundamental goals.

In a situation of transient difficulty, where the patient needs to survive a crisis (divorce, bereavement, physical disease, etc.) without mental breakdown, or where mental breakdown has occurred, and supportive therapy aims to minimise the likelihood of recurrence, change of values, defences, or behaviour may be quite undesirable, and support frequently aims to bolster familiar coping mechanisms.

Where a presented problem involves longstanding focal or general inadequacy of life-strategy, change may be the principal goal of therapy, and its achievement may require the dismantling of familiar coping mechanisms, construed neurotic or maladaptive. Dynamic psychotherapy requires the abandonment of safe havens behind neurotic defences, for the more challenging and dangerous pattern of less defensive relating, with the reality of regression and risk of breakdown.

I believe, with Dr Crown, P. Sifneos and others, that dynamic psychotherapy carries the likelihood of worsening adaptation before improvement occurs, based on more adaptive life strategies emerging.

Dr Crown concludes that supportive is not psychotherapy and vice versa. He does not concede here, although the abstract of his 1986 talk did concede, that supportive therapy may be therapeutic. Within a definition of psychotherapy which includes substantial personal change as an essential ingredient, I believe his latest analysis to be accurate. However, in the interests of a catholic and tolerant defnition of psychotherapy, I would be much happier to accentuate the therapeutic construction of supportive therapy. Many psychotherapists provide support for numbers of their patients, and many other professionals view their supportive endeavours as therapeutic. Applying a medical model of therapy and examining 'fit', there are clearly 'curative' and 'palliative' categories of intervention, each deemed 'therapeutic'. Some psychotherapists would possibly argue that palliation is not part of their remit; I believe that to be an untenable posture.

It has to be conceded that dynamic and supportive therapies differ. Radical and conservative managements always do, but each can justifiably claim to be therapeutic. If it were conceded that supportive therapy be therapeutic but not psychotherapy, the question would need to be addressed; if not the psyche, then what is being treated?

I can supply no satisfactory solution to this dilemma and accordingly I cast my vote in favour of accepting supportive psychotherapy as a kind of psychotherapy, albeit conservative and limited in its goals.

This view would not concede the status 'psychotherapist' to practitioners of supportive therapy not engaged in, trained in, and committed to radical curative forms of psychotherapeutic endeavour. While this debate (who is a psychotherapist?) is probably nearing, if not conclusion, at least significant progress, it would be a pity for the established forms of radical psychotherapy to regard as a challenge, or with any trepidation, any pretensions to status which may be claimed for supportive psychotherapy on behalf of its many non-psychotherapist practitioners.

I believe an emerging profession of 'psychotherapist' will have no difficulty identifying practitioners of radical intervention, with acceptable 